\title{
Three tumor markers for improved efficacy in the management of patients with malignant pleural mesothelioma
}

\author{
Akifumi Nakamura ${ }^{1}$, Teruhisa Takuwa ${ }^{1}$, Masaki Hashimoto ${ }^{1}$, Ayumi Kuroda ${ }^{1}$, Toru Nakamichi ${ }^{1}$, \\ Seiji Matsumoto $^{1}$, Nobuyuki Kondo ${ }^{1}$, Takashi Kijima ${ }^{2}$, Seiki Hasegawa ${ }^{1}$ \\ ${ }^{1}$ Division of Thoracic Surgery, Department of Surgery, Hyogo College of Medicine, Nishinomiya, Japan; ${ }^{2}$ Division of Respiratory Medicine, \\ Department of Internal Medicine, Hyogo College of Medicine, Nishinomiya, Japan \\ Contributions: (I) Conception and design: A Nakamura; (II) Administrative support: S Hasegawa; (III) Provision of study materials or patients: A \\ Nakamura; (IV) Collection and assembly of data; A Nakamura; (V) Data analysis and interpretation: A Nakamura; (VI) Manuscript writing: All \\ authors; (VII) Final approval of manuscript: All authors. \\ Correspondence to: Akifumi Nakamura. Division of Thoracic Surgery, Department of Surgery, Hyogo College of Medicine, 1-1, Mukogawa-cho, \\ Nishinomiya 663-8501, Japan. Email: xxmp97511@zeus.eonet.ne.jp.
}

Background: Evaluation of tumor markers may facilitate follow-up of malignant pleural mesothelioma (MPM). We aimed was to evaluate the value of tumor markers for monitoring and predicting recurrence in patients with MPM.

Methods: In total, 152 patients who underwent curative-intent surgery after induction chemotherapy for MPM between July 2004 and December 2017 were retrospectively reviewed. Preoperative and postoperative ( $\leq 3$ months after surgery) levels of soluble mesothelin-related peptide (SMRP), cytokeratin 19 fragment (Cyfra21-1), and tissue polypeptide antigen (TPA) and rates of recurrence and non-recurrence were evaluated. Factors associated with recurrence-free survival (RFS) were assessed using the Kaplan-Meier method and Cox proportional hazards model.

Results: Of the 152 patients, the positive rates of preoperative SMRP, Cyfra21-1, and TPA, levels were $26.7 \%, 8.6 \%, 9.6 \%$, respectively; the respective postoperative levels were $4.0 \%, 6.3 \%$, and $6.5 \%$; the respective levels in patients with recurrence were $39.3 \%, 31.4 \%, 28.6 \%$; the respective levels in patients with no recurrence were $3.7 \%, 0.0 \%, 3.8 \%$. Nearly half $(45.2 \%)$ of the patients with recurrence exhibited an increase in one or more tumor marker levels. Multivariate analysis revealed that the preoperative positive rates of one or more of the three tumor markers (hazard ratio: 1.8, 95\% confidence interval: $1.1-2.8 ; \mathrm{P}=0.02$ ) were independent significant predictors of recurrence.

Conclusions: The positive rates of SMRP, Cyfra21-1, and TPA in recurrence-free patients were extremely low, with high specificity. Preoperative levels of SMRP, Cyfra21-1, and TPA, which identified patients with a high risk for recurrence, could improve management of patients with MPM.

Keywords: Malignant pleural mesothelioma (MPM); tumor markers; soluble mesothelin-related peptide (SMRP); cytokeratin 19 fragment (Cyfra21-1); tissue polypeptide antigen (TPA)

Submitted May 12, 2020. Accepted for publication Sep 24, 2020.

doi: $10.21037 /$ jtd-20-1910

View this article at: http://dx.doi.org/10.21037/jtd-20-1910

\section{Introduction}

Malignant pleural mesothelioma (MPM) is a rare, aggressive tumor of the pleura, with a recurrence rate post treatment. Baldini et al. reported failure after multimodal therapy for MPM. Of the 158 evaluable patients, MPM recurred in
118 (75\%) patients (1). To date, the follow-up of patients with MPM after treatment remains challenging. Evaluation of postoperative recurrence in MPM is typically based on radiological examination. However, in clinical practice, such evaluation is sometimes challenging because of the unique 

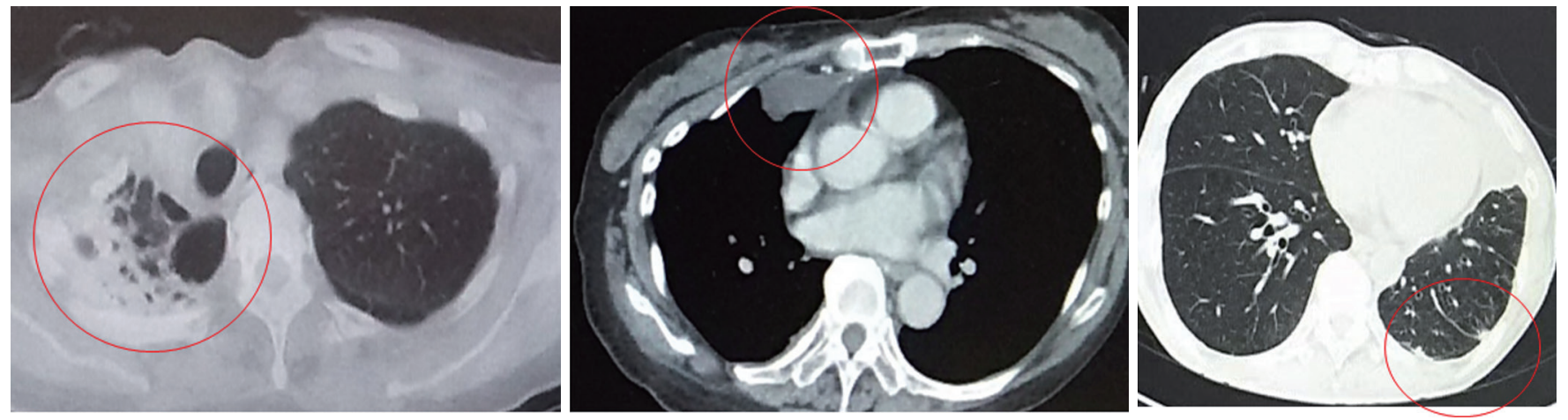

Figure 1 Postoperative changes. Computed tomographic (CT) images showing postoperative changes, but not recurrence. Radiographic diagnosis of recurrence can be difficult in postoperative chest CT because of postoperative changes.

growth pattern of MPM and the postoperative changes after curative-intent surgery, including pleurectomy/ decortication (P/D) or extrapleural pneumonectomy (EPP) (Figure 1). In this context, evaluation of tumor markers for MPM will help provide insight into appropriate followup of patients with MPM for recurrence. Several recent studies have addressed the efficacy of certain tumor markers for MPM. Wheatley-Price et al. reported that the soluble mesothelin-related peptide (SMRP) is a potentially useful marker of the disease course (2). Scuouwink et al. reported that cytokeratin 19 fragment (Cyfra 21-1) and tissue polypeptide antigen (TPA) demonstrated a prognostic value in patients with MPM (3). However, research to evaluate tumor markers in the postoperative follow-up of patients with MPM is scarce. In this study, we aimed to determine the usefulness of SMRP, Cyfra21-1, and TPA as tumor markers for prediction and postoperative monitoring of recurrence in patients with MPM.

\section{Methods}

\section{Patients and data}

A total of 152 consecutive patients who received curativeintent surgery after induction chemotherapy for MPM between July 2004 and December 2017 at Hyogo College of Medicine Hospital (Nishinomiya, Hyogo, Japan) were included. Patients who received exploratory thoracotomy were excluded. This study was a retrospective review of medical records from a prospectively registered database. The study was conducted in accordance with the Reporting Recommendations for Tumor Marker Prognostic Studies (REMARK) reporting checklist (4) (available at http:// dx.doi.org/10.21037/jtd-20-1910).

\section{Multimodality treatment protocol}

The multimodality treatment was performed according to our previous reports (5-8). The treatment protocol is presented in Figure 2. The eligibility criteria were as follows: age $\leq 80$ years, histologically confirmed diagnosis of any type of MPM, clinical stage T1-3N0-1M0 disease (IMIG $8^{\text {th }}$ edition), performance status of $0-1$, and no major comorbidities. Patients with sarcomatoid histology were excluded from our multimodality treatment protocol.

All patients received induction chemotherapy before curative-intent surgery. Induction chemotherapy comprised 3 cycles of platinum-based doublet chemotherapy. After the induction chemotherapy, tumor response was assessed by computed tomography $(\mathrm{CT})$ and $\left({ }^{18} \mathrm{~F}\right)$-fluorodeoxyD-glucose positron-emission tomography (FDG-PET)/ CT using a modified version of the Response Evaluation Criteria in Solid Tumors (mRECIST) (9). The cancer board comprised pulmonologists, thoracic surgeons, radiologists, and pathologists who evaluated the treatment response and determined the treatment plan. Curative-intent surgery was planned in patients who achieved more than stable disease according to the mRECIST criteria.

Curative-intent surgery (EPP or P/D) was performed in the above patients 4-8 weeks after induction chemotherapy completion. Our policy regarding curative-intent surgery is to achieve macroscopic complete resection (MCR). Before September 2012, EPP was selected. P/D was introduced in September 2012 at our institution and has been increasingly performed. Conversion from P/D to EPP was decided only when diffuse tumor invasion to the pulmonary parenchyma was found during surgery. Adjuvant high-dose hemithoracic irradiation, using either three-dimensional conformal or intensity-modulated radiotherapy, was performed within 


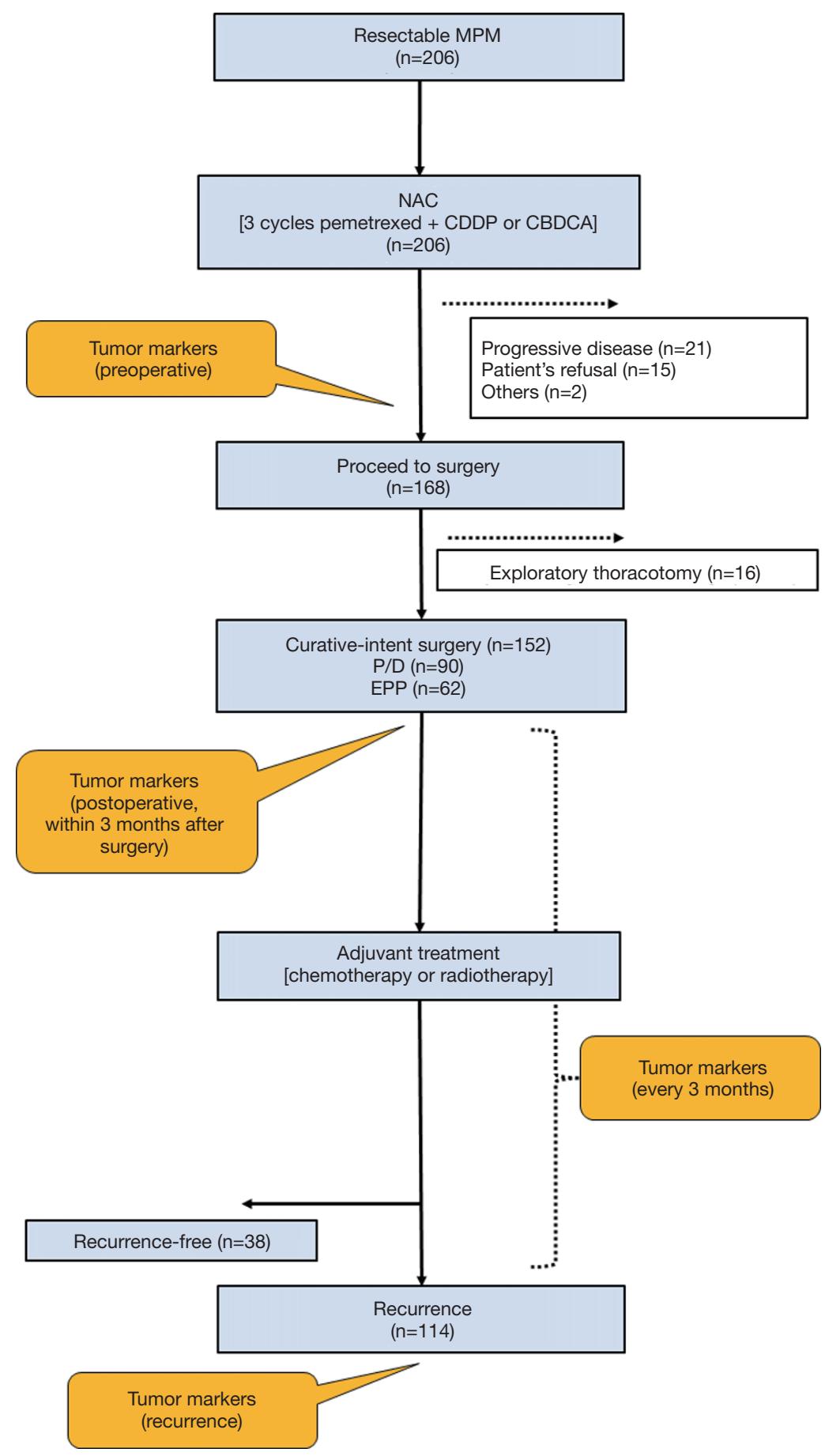

Figure 2 Treatment course and tumor markers. The treatment course comprised neoadjuvant chemotherapy, followed by curative-intent surgery and adjuvant chemotherapy or radiotherapy. SMRP, Cyfra21-1, and TPA were examined preoperatively, postoperatively (every 3 months), and at the time of recurrence. We evaluated SMRP, Cyfra 21-1, and TPA preoperatively, postoperatively (within 3 months after curative-intent surgery), and at the time of recurrence or determination of non-recurrence (based on the updated data in the follow-up period if patients did not develop recurrence). MPM, malignant pleural mesothelioma; NAC, neoadjuvant chemotherapy; CDDP, cisplatin; CBDCA, carboplatin; P/D, pleurectomy/decortication; SMRP, soluble mesothelin-related peptide; Cyfra21-1, cytokeratin 19 fragment; TPA, tissue polypeptide antigen. 
12 weeks of EPP. A total dose of 54 Gy was delivered in 30 fractions at $1.8 \mathrm{~Gy} /$ day. Adjuvant chemotherapy was performed within 8-12 weeks of P/D. Most patients received platinum-based doublet chemotherapy. Completion of adjuvant chemotherapy was defined as having completed $\geq 2$ cycles.

\section{Postoperative follow-up}

Clinical data, including patient characteristics, surgical reports, radiological findings, histopathology, and survival, were collected by reviewing charts. All patients underwent physical and blood examinations for tumor markers, as well as CT imaging every 3 months at our outpatient treatment facility. Further detailed examinations, including contrastenhanced CT or FDG-PET/CT, were performed if the primary CT revealed symptoms suggestive of recurrence, such as "pleural thickening", "mass", or "lymphadenopathy", or if the primary CT was positive for the tumor markers. During the follow-up, our cancer board recommended that the patients be monitored for signs of MPM recurrence by physical examination, radiological findings, blood examination, and pathological findings. Biopsy for tumor recurrence was performed as needed. The recurrence diagnosis was determined by the cancer board.

Local recurrence was defined as tumor relapse in the ipsilateral hemithorax or in the mediastinum, and distant recurrence was defined as tumor recurrence in the contralateral hemithorax, abdomen, or another extrathoracic location.

\section{Tumor markers}

Serum biomarker levels were measured as follows. Serum was separated immediately from blood samples collected from patients and was stored at $-80^{\circ} \mathrm{C}$. The serum SMRP levels were measured using a chemiluminescent enzyme immunoassay (Fujirebio Inc., Tokyo, Japan), according to the manufacturer's instructions. The serum Cyfra21-1 and TPA levels were measured using commercially available immunoassay systems, according to the manufacturers' instructions: Serum Cyfra21-1 levels were determined using a chemiluminescent enzyme immunoassay (Fujirebio Inc.) and serum TPA levels using a chemiluminescent immunoassay (DiaSorin Inc., Saluggia VC, Italy). These tumor markers were examined preoperatively, postoperatively (every 3 months), and at the time of recurrence (Figure 2). The following cutoff values for reference versus elevated levels were used based on previous reports: SMRP, $1.5 \mathrm{nmol} / \mathrm{L}$; Cyfra21-1, $3.5 \mathrm{ng} / \mathrm{mL}$; and TPA, 75 U/L. We evaluated SMRP, Cyfra21-1, and TPA preoperatively, postoperatively, at the time of recurrence, and at recurrence-free determination, considering these three tumor markers as expressed (positive) or not expressed (negative). Postoperative was defined as within 3 months after curative-intent surgery. The quantification of tumor markers in patients with recurrence-free survival (RFS) was defined as based on updated data during the follow-up period.

\section{Statistical analysis}

The positive rate of each tumor marker was calculated preoperatively, postoperatively (within 3 months after curative-intent surgery), at the time of recurrence, and at the time of recurrence-free determination. RFS was estimated using the Kaplan-Meier method, and univariate prognostic analysis was performed using the log-rank test. The Cox proportional hazards model was used for multivariate analysis, which included significant clinical variables. All analyses were performed with JMP 14 (SAS Institute Inc., Cary, NC, USA). Differences were considered to be statistically significant for $\mathrm{P}$ values of $<0.05$.

\section{Ethics}

This study complied with the standards of the Declaration of Helsinki (as revised in 2013). All eligible patients were included after they provided informed consent. The institutional review board at the Hyogo College of Medicine (No. 3280) approved the study on August 1, 2019.

\section{Results}

\section{Patient characteristics}

Between July 2004 and December 2017, a total of 206 consecutive patients with MPM were eligible for multimodality treatment at our institution (Table 1, Figure 2). All patients received neoadjuvant chemotherapy. Of these, 168 patients proceeded to surgery, and the remaining 38 patients did not proceed to surgery because of progressive disease $(n=21)$, patient refusal $(n=15)$, and other reasons $(\mathrm{n}=2)$. Of the 168 patients who proceeded to surgery, 16 received exploratory thoracotomy. Finally, 152 (men, 128) patients received curative-intent surgery for MPM. 
The median age of the patients at the curative-intent surgery was 64 years (range, 16-79 years), and 90 (59.2\%) patients were treated with P/D and 62 (40.8\%) with EPP. Histological assessment revealed the final pathology as epithelioid for $143(94.1 \%)$ patients and non-epithelioid for $9(5.9 \%)$ patients. According to the pathological stage of the International Mesothelioma Interest Group staging system (Version 8), 35 patients had stage IA, 58 stage IB, 12 stage II, 37 stage IIIA, 9 stage IIIB, and 1 stage IV disease. MCR was achieved in 145/152 (95.4\%) of the patients. Finally, $119(78.3 \%)$ patients completed surgery plus both neoadjuvant and adjuvant treatments.

\section{RFS and tumor marker analysis}

Of the 152 patients, 114 (75.0\%) developed recurrence. In this study, the median follow-up period was 27 months, and the median time to recurrence was 19.0 months. The 3 -year RFS rate was $27.7 \%$. The sites of first recurrence were local (ipsilateral hemithorax or the mediastinum) in 65 patients and distant (abdomen or contralateral hemithorax, brain, bone, and so on) in 26 patients. Both local and distant recurrence occurred in 23 patients.

Figure 3 presents the details of the tumor markers. The positive rates of preoperative tumor marker levels in the patients were as follows: SMRP, 26.7\%; Cyfra21-1, $8.6 \%$; and TPA, $9.6 \%$. Most patients were negative for postoperative tumor markers. The positive rates of postoperative tumor marker levels in the patients were as follows: SMRP, 4.0\%; Cyfra21-1, 6.3\%; and TPA, 6.5\%. The positive rates of tumor marker levels in patients at the time of recurrence diagnosis were as follows: SMRP, 39.3\%; Cyfra 21-1, 31.4\%; and TPA, 28.6\%. Nearly half (45.2\%) of the patients with recurrence exhibited an increase in one or more tumor marker levels.

Figure 4 presents the tumor marker details grouped by the recurrence pattern. The positive rates of tumor marker levels in patients with only local recurrence were as follows: SMRP, 39.5\%; Cyfra21-1, 19.6\%; and TPA, 16.7\%. The positive rates of tumor marker levels in patients with only distant recurrence were as follows: SMRP, $30.0 \%$; Cyfra21-1, 37.5\%; TPA, and 34.8\%. The positive rates of tumor marker levels in patients with both local and distant recurrence were as follows: SMRP, 46.2\%; Cyfra21-1, $54.5 \%$; and TPA, $52.4 \%$ (Figure 4).

By contrast, the positive rates of tumor marker levels in recurrence-free patients were as follows: SMRP, 3.7\%; Cyfra21-1, 0.0\%; and TPA, 3.8\% (Figure 5).
Table 1 Clinical characteristics of the patients

\begin{tabular}{|c|c|c|}
\hline Characteristic & Number $(\mathrm{N}=152)$ & $(\%)$ \\
\hline Age, median (years) & 64 & \\
\hline \multicolumn{3}{|l|}{ Sex } \\
\hline Male & 128 & 84.2 \\
\hline Female & 24 & \\
\hline \multicolumn{3}{|l|}{ Pathology } \\
\hline Epithelioid & 143 & 94.1 \\
\hline Non-epithelioid & 9 & \\
\hline \multicolumn{3}{|l|}{ Pathological Stage ( $8^{\text {th }}$ edition) } \\
\hline IA & 35 & 23.0 \\
\hline IB & 58 & 38.2 \\
\hline II & 12 & 7.9 \\
\hline IIIA & 37 & 24.3 \\
\hline IIIB & 9 & 5.9 \\
\hline IV & 1 & 0.7 \\
\hline \multicolumn{3}{|l|}{ Surgery } \\
\hline$P / D$ & 90 & 59.2 \\
\hline EPP & 62 & \\
\hline Macroscopic complete resection & 145 & 95.4 \\
\hline Completed multimodal therapy & 119 & 78.3 \\
\hline Recurrence & 114 & 75.0 \\
\hline Local & 65 & \\
\hline Distant & 26 & \\
\hline Both (local + distant) & 23 & 84.2 \\
\hline
\end{tabular}

\section{Univariate and multivariate analyses}

Univariate prognostic analysis based on Kaplan-Meier curves revealed that RFS was significantly associated with histology, pathological stage, and the preoperative positive rates of the three tumor marker levels (lower) (Table 2). RFS was significantly longer in patients who were preoperatively negative for all the three tumor markers (SMRP, Cyfra 21-1, and TPA) than those who were preoperatively positive for one or more of the three tumor markers (median RFS: 20.7 vs. 10.7 months, $\mathrm{P}=0.03$ ), those with pathological stage IA disease than those with pathological stage IB, II, IIIA, or IIIB disease (median RFS: 36.8 vs. 16.3 months, $\mathrm{P}<0.001$ ), and those with epithelioid tumors than those with non- 

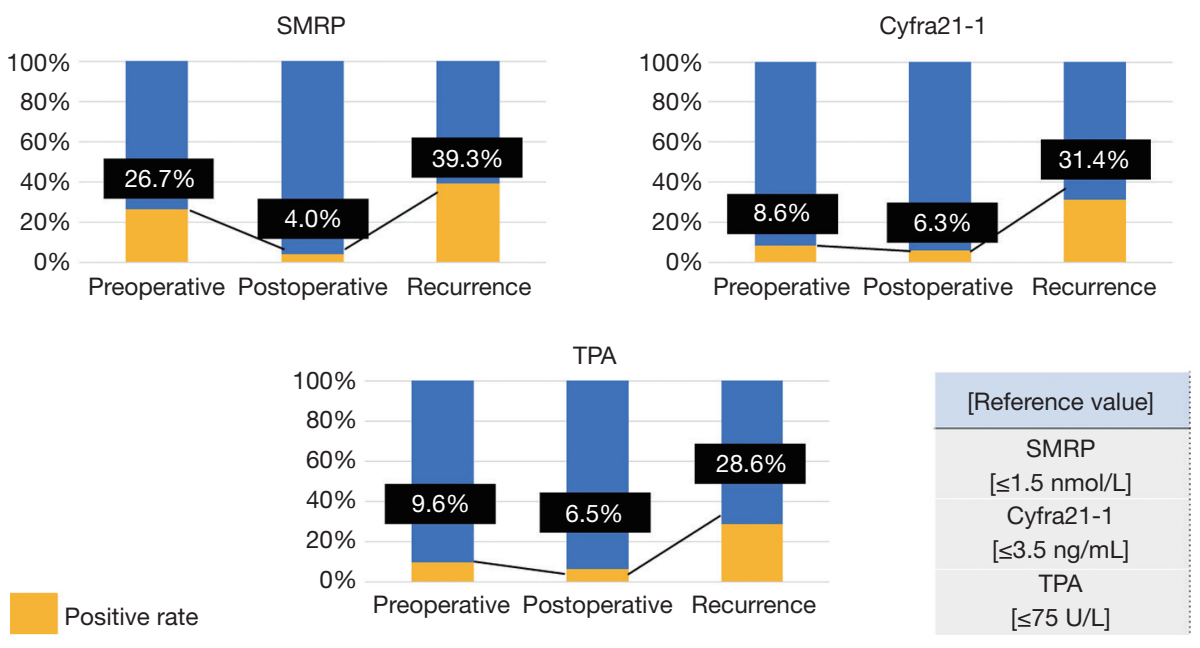

Figure 3 Tumor marker analysis. The preoperative positive rates of tumor markers ranged from $10 \%$ to $20 \%$. When radiographic findings diagnosed recurrence in patients, the positive rates of the tumor markers ranged from $30 \%$ to $40 \%$. SMRP, soluble mesothelin-related peptide; Cyfra21-1, cytokeratin 19 fragment; TPA, tissue polypeptide antigen.
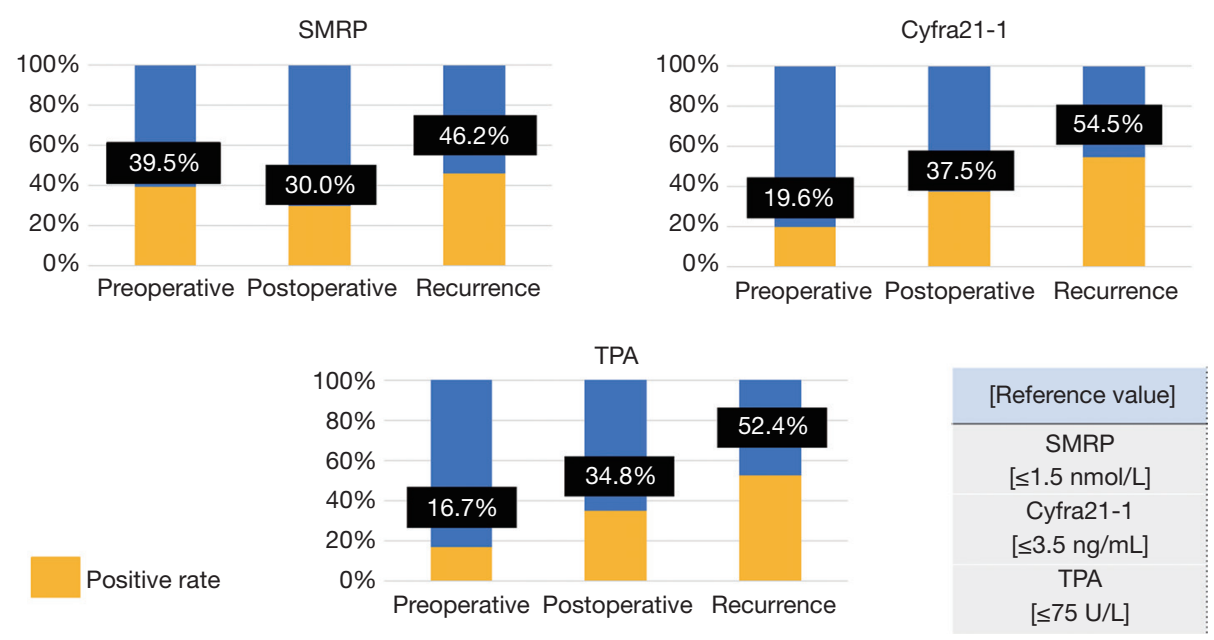

Figure 4 Tumor marker analysis according to recurrence pattern. Tumor markers are grouped according to recurrence pattern. The positive rates of tumor markers in patients with only local or distant recurrence ranged from $15 \%$ to $40 \%$. The rate for patients with both local and distant recurrence was 50\%. SMRP, soluble mesothelin-related peptide; Cyfra21-1, cytokeratin 19 fragment; TPA, tissue polypeptide antigen.

epithelioid tumors (median RFS: 19.6 vs. 6.3 months, $\mathrm{P}<0.001)$. Multivariate analysis using the Cox proportional hazards model for post-recurrence survival revealed that RFS was independently predicted by histology [hazard ratio (HR): 3.0, 95\% confidence interval (CI): 1.4-6.3; $\mathrm{P}=0.004]$, pathological stage (HR: 2.3, 95\% CI: 1.4-3.9, $\mathrm{P}<0.001$ ), and preoperative positive rates of three tumor marker levels (lower) (HR: 1.8, 95\% CI: 1.1-2.8; P=0.02) (Table 3).

\section{Discussion}

Two important findings in this study support the use of SMRP, Cyfra21-1, and TPA as tumor markers for improved efficacy in the management of patients with MPM. First, the positive rates of these tumor marker serum levels in recurrence-free patients were extremely low. The specificity was high, and these markers were found to be useful during follow-up monitoring. If any one of these markers was 


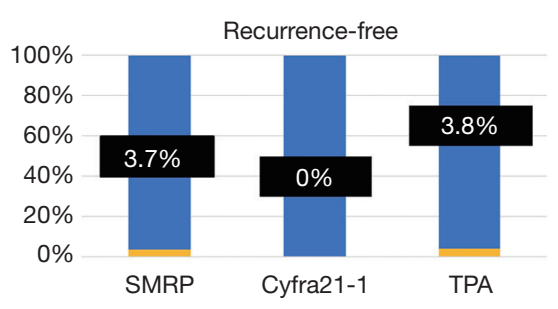

Positive rate

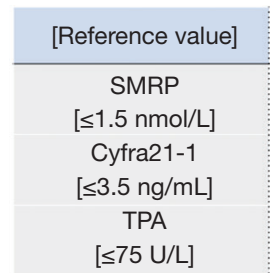

Figure 5 Tumor marker analysis in recurrence-free patients. The positive rates of tumor marker levels in recurrence-free patients were as follows: SMRP, 3.7\%; Cyfra21-1, 0\%; and TPA, 3.8\%. SMRP, soluble mesothelin-related peptide; Cyfra21-1, cytokeratin 19 fragment; TPA, tissue polypeptide antigen.

positive, there was a high probability of recurrence and, therefore, a need for more detailed examinations. Second, one or more preoperative positive rates of these marker levels were significant risk factors for recurrence in the multivariate analysis of patients with non-epithelioid, nonStage IA tumors, indicating that these markers can be used to distinguish patients likely to develop recurrence from those not likely to develop recurrence. If a patient was positive for one or more of the tumor markers preoperatively, the probability of recurrence was much higher than the probability of recurrence in patients with low marker levels.

It is well known that MPM recurs in most patients after treatment, and the follow-up of patients with MPM after treatment remains a challenge. We previously reported post-recurrence chemotherapy for patients with MPM undergoing EPP or P/D $(6,8)$. In these studies, the survival rate in post-recurrence chemotherapy group was than that in the no post-chemotherapy group. In our hospital, follow-up for MPM recurrence after multimodality therapy is performed every $2-3$ months, which helps us detect early recurrence (the earlier, the better), because these patients are in generally good condition. Early diagnosis of recurrent MPM is imperative. However, the diagnosis of postoperative recurrence in MPM is sometimes refractory because of the unique growth pattern as a pleural ring rather as a discrete mass of MPM and other postoperative changes. These postoperative changes can be difficult to discern by anatomical standards by performing CT. Therefore, FDG$\mathrm{PET} / \mathrm{CT}$ can be used but is limited by false-negative results caused by postoperative hypermetabolism (10). Even in such cases, the three tumor markers evaluated in this study could be useful in follow-up for MPM, because the positive rates of these markers in recurrence-free patients were low. During postoperative follow-up, if serum results for any one of these markers were positive, the probability of recurrence was high. In addition, the combination of these tumor markers could improve the management efficacy of patients with MPM. In this study, $45.2 \%$ of the patients with recurrence exhibited an increase in one or more of these marker levels. Cristaudo et al. reported that the combination of multiple markers can be highly useful to increase sensitivity and specificity in monitoring patients with MPM (11).

In this study, we investigated the use of preoperative tumor markers for predicting recurrence in patients with MPM. The positive rates of preoperative tumor marker levels in these patients were as follows: SMRP, 26.7\%; Cyfra21-1, 8.6\%; and TPA, 9.6\%, with low sensitivity. Although several tumor markers have been suggested as diagnostic tools for MPM (12), SMRP remains the most efficient tumor marker $(10,13,14)$. Hollevoet et al. reported the diagnostic accuracy and use of SMRP in early diagnosis and performed an individual patient data meta-analysis. Of 1,026 patients, the sensitivities and specificities of SMRP in the different studies ranged widely from 19-68\% and $88-100 \%$, respectively (15). This heterogeneity can be explained by differences in study populations. The tumor size (I or II vs. III or IV) and histological subtype (epithelioid, sarcomatoid, or biphasic) significantly affected the diagnostic accuracy of SMRP. The highest area under the curve was observed for differentiating patients with epithelioid stage III or IV MPM. By contrast, Scuouwink et al. reported that at the time of diagnosis, Cyfra 21-1 and TPA were elevated at $50 \%$ and $58 \%$, respectively (3). However, in our study, both preoperative and recurrence 
Table 2 Univariate analysis using the Kaplan-Meier method for recurrence after surgery

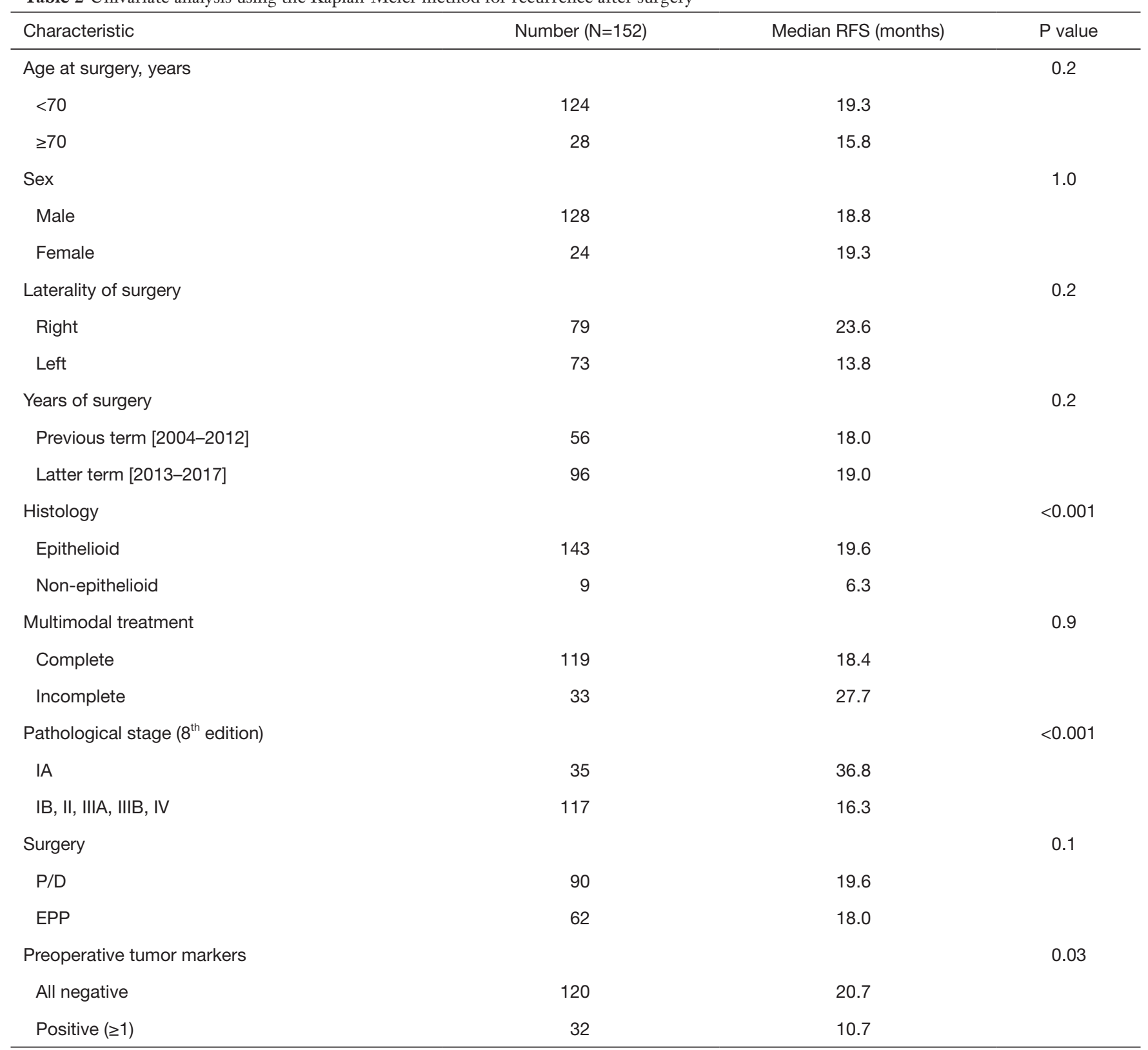

RFS, recurrence-free survival; P/D, pleurectomy/decortication; EPP, extrapleural pneumonectomy.

positive rates of tumor markers were low, which could be attributed to the following reasons. First, most patients were in the early stage of the disease. Most patients (105/152; 69.1\%) had pathological stage I + II ( $8^{\text {th }}$ edition $)$ disease. Second, we were able to detect early recurrence because of careful follow-up (every $2-3$ months) after multimodality therapy. Taken together, these results can be attributed to the fact that tumor volumes at the time of diagnosis and recurrence were low. In fact, the positive rates of tumor marker levels in patients with both local and distant recurrence were higher than those in patients with only local or distant recurrence.

To elucidate the mechanism of risk factors for recurrence, multivariate analysis was performed. The analysis revealed that histology, pathological stage, and positive $(\geq 1)$ of preoperative tumor markers were independent factors 
Table 3 Multivariate analysis using a Cox proportional hazards model for recurrence-free survival after surgery

\begin{tabular}{lcc}
\hline Factor & P value & HR (95\% Cl) \\
\hline Histology, non-epithelioid & 0.004 & $3.0(1.4-6.3)$ \\
Pathological Stage, IB, II, IIIA, IIIB, IV & $<0.001$ & $2.3(1.4-3.9)$ \\
Preoperative tumor markers, positive ( $\geq 1)$ & 0.020 & $1.8(1.1-2.8)$ \\
\hline
\end{tabular}

$\mathrm{HR}$, hazard ratio; $\mathrm{Cl}$, confidence interval.

associated with RFS. Several studies have reported that patients with non-epithelioid and advanced-stage tumors had poor prognosis, although this finding is obvious to a certain extent. However, to the best of our knowledge, detailed studies investigating the effects of preoperative tumor markers on RFS are sparse. In this study, multivariate analysis showed an association between positivity for the three tumor markers and recurrence, with a substantially greater number of patients. These tumor markers remained significant risk factors after correction for histological and pathological stage. Occasionally, there have been reports of preoperative SMRP being associated with survival outcomes in MPM. Tian et al. performed a meta-analysis to evaluate the prognostic value of preoperative SMRP and the effect of clinicopathological characteristics on the survival of patients with MPM. Of eight eligible studies involving 579 patients, preoperative SMRP level was significantly associated with survival in patients with MPM (HR: 1.958, 95\% CI: 1.5-2.5, $\mathrm{P}<0.001)(16)$. By contrast, limited data exist on the effects of Cyfra 21-1 and TPA on survival outcomes in MPM. Scuouwink et al. retrospectively investigated preoperative Cyfra 21-1 and TPA for their significance in predicting survival in 52 patients with MPM. Preoperative Cyfra 21-1 and TPA were independent prognostic factors, along with performance status and platelet count, in their multivariate analysis. In our study, preoperative positivity for one or more of the three tumor markers in patients with MPM was a significant risk factor for recurrence in the multivariate analysis.

\section{Limitations}

This study is not without limitations. The long-term and retrospective single-center design portrays a risk of bias. The postoperative tumor marker levels were not measured with a standardized schedule. The tumor markers were not measured at the time of diagnosis, which might have affected the results. The Effects of the analysis in individual patients over time remain to be evaluated. Therefore, the results should be carefully interpreted. The prevalence rate of the three markers in the patients who experienced a recurrence was not too high (45.2\%). This suggests the low sensitivity of the test. We did not analyze change in the level of biomarker expression over time. Further study is required to examine the percentage change in tumor marker levels. In addition, in this study, patients were followed up every 3-6 months by performing physical examination, blood testing, CT, and FDG-PET/CT. However, this followup protocol may not be possible in all countries because of differences in healthcare systems. In most patients, recurrence is diagnosed on the basis of radiographic or pathological findings. Tumor markers play a supplementary role at this time.

\section{Conclusions}

The positive rates of the tumor markers SMRP, Cyfra21-1, and TPA were low in recurrence-free patients, with high specificity. In multivariate analysis, preoperative positivity for one or more of these markers was a significant risk factor for recurrence. Thus, the combination of these tumor markers could enhance the management efficacy of patients with MPM.

\section{Acknowledgments}

Funding: None.

\section{Footnote}

Reporting Checklist: The authors have completed the Reporting Recommendations for Tumor Marker Prognostic Studies (REMARK) reporting checklist. Available at http:// dx.doi.org/10.21037/jtd-20-1910

Data Sharing Statement: Available at http://dx.doi. org/10.21037/jtd-20-1910

Conflicts of Interest: All authors have completed the ICMJE 
uniform disclosure form (available at http://dx.doi. org/10.21037/jtd-20-1910). The authors have no conflicts of interest to declare.

Ethical Statement: The authors are accountable for all aspects of the work in ensuring that questions related to the accuracy or integrity of any part of the work are appropriately investigated and resolved. Informed consent was obtained from all eligible patients included in the program. This study complied with the standards of the Declaration of Helsinki (as revised in 2013). All eligible patients were included after they provided informed consent. The institutional review board at the Hyogo College of Medicine (No. 3280) approved the study on August 1, 2019.

Open Access Statement: This is an Open Access article distributed in accordance with the Creative Commons Attribution-NonCommercial-NoDerivs 4.0 International License (CC BY-NC-ND 4.0), which permits the noncommercial replication and distribution of the article with the strict proviso that no changes or edits are made and the original work is properly cited (including links to both the formal publication through the relevant DOI and the license). See: https://creativecommons.org/licenses/by-nc-nd/4.0/.

\section{References}

1. Baldini EH, Richards WG, Gill RR, et al. Updated patterns of failure after multimodality therapy for malignant pleural mesothelioma. J Thorac Cardiovasc Surg 2015;149:1374-81.

2. Wheatley-Price P, Yang B, Patsios D, et al. Soluble mesothelin-related Peptide and osteopontin as markers of response in malignant mesothelioma. J Clin Oncol 2010;28:3316-22.

3. Schouwink H, Korse CM, Bonfrer JM, et al. Prognostic value of the serum tumour markers Cyfra 21-1 and tissue polypeptide antigen in malignant mesothelioma. Lung Cancer 1999;25:25-32.

4. McShane LM, Altman DG, Sauerbrei W, et al. REporting recommendations for tumour MARKer prognostic studies (REMARK). Br J Cancer 2005;93:387-91.

5. Hasegawa S, Kondo N, Matsumoto S, et al. Surgical Risk and Survival Associated With Less Invasive Surgery for Malignant Pleural Mesothelioma. Semin Thorac Cardiovasc Surg 2019;31:301-9.
6. Takuwa T, Hashimoto M, Matsumoto S, et al. Postrecurrence chemotherapy for mesothelioma patients undergoing extrapleural pneumonectomy. Int J Clin Oncol 2017;22:857-64.

7. Hashimoto M, Takeuchi J, Takuwa T, et al. Pleural thickness after neoadjuvant chemotherapy is a prognostic factor in malignant pleural mesothelioma. J Thorac Cardiovasc Surg 2019;157:404-13.

8. Nakamura A, Takuwa T, Hashimoto M, et al. Clinical outcomes with recurrence after pleurectomy/decortication for malignant pleural mesothelioma. Ann Thorac Surg 2020;109:1537-43.

9. Byrne MJ, Nowak AK. Modified RECIST criteria for assessment of response in malignant pleural mesothelioma. Ann Oncol 2004;15:257-60.

10. Burt BM, Lee HS, Lenge De Rosen V, et al. Soluble Mesothelin-Related Peptides to Monitor Recurrence After Resection of Pleural Mesothelioma. Ann Thorac Surg 2017;104:1679-87.

11. Cristaudo A, Bonotti A, Guglielmi G, et al. Serum mesothelin and other biomarkers: what have we learned in the last decade? J Thorac dis 2018;10:S353-59.

12. Arnold DT, De Fonseka D, Hamilton FW. et al. Prognostication and monitoring of mesothelioma using biomarkers: a systematic review. Br J Cancer 2017;116:731-41.

13. Robinson BW, Creaney J, Lake R, et al. Soluble mesothelin-related protein--a blood test for mesothelioma. Lung Cancer 2005:49:S109-11.

14. Fukuoka K, Kuribayashi K, Yamada S et al. Combined serum mesothelin and carcinoembryonic antigen measurement in the diagnosis of malignant mesothelioma. Mol Clin Oncol 2013;1:942-48.

15. Hollevoet K, Reitsma JB, Creaney J, et al. Serum mesothelin for diagnosing malignant pleural mesothelioma: an individual patient data meta-analysis. J Clin Oncol 2012;30:1541-49.

16. Tian L, Zeng R, Wang X, et al. Prognostic significance of soluble mesothelin in malignant pleural mesothelioma: a meta-analysis. Oncotarget 2017;8:46425-35.

Cite this article as: Nakamura A, Takuwa T, Hashimoto $M$, Kuroda A, Nakamichi T, Matsumoto S, Kondo N, Kijima T, Hasegawa S. Three tumor markers for improved efficacy in the management of patients with malignant pleural mesothelioma. J Thorac Dis 2020;12(11):6712-6721. doi: 10.21037/jtd-20-1910 